\title{
Color coding of spatial frequencies using incoherent optical processing
}

\author{
G. Indebetouw
}

\begin{abstract}
An incoherent optical processing technique for color coding the spatial frequency content of an input is described. Experimental results and comparison with the output of a coherent filtering system are discussed. Possible applications such as texture recognition in aerial imagery or radiography analysis are suggested.
\end{abstract}

\section{Introduction}

The use of color in image processing has, in the last few years, received considerable attention. Adding color to the output of an optical processor, either to enhance detail or contrast ... . etc., very often leads to an output that is more accurately readable, qualitatively simpler to analyze, and altogether visually more pleasant.

A great number of pseudocolor coding schemes have been proposed. Most of them belong to two classes concerned with either the color coding of gray levels (equidensitometry) or the color coding of spatial frequencies. Density slicing and coloring are a nonlinear operation that can be performed by encoding the density slices with a specially prepared halftone screen and decoding them in a coherent ${ }^{1}$ or incoherent ${ }^{2-4}$ spatial filtering setup.

Most of the techniques proposed for the color coding of spatial frequencies use holographic techniques. They take advantage of the diffraction phenomena that occur in, for example, the white light reconstruction of rainbow holograms. ${ }^{5-7}$ These techniques require either a multiwavelength laser for recording the hologram or the superposition of multistage copies of several holograms. A much simpler approach using colored spatial filters in the Fourier plane of a partially coherent processor has also been demonstrated. ${ }^{8}$

This paper describes the results obtained by using an incoherent optical filtering technique to color code the spatial frequency content of an input picture. The

The author is with Virginia Polytechnic Institute \& State University, Physics Department, Blacksburg, Virginia 24060.

Received 23 August 1979.

0003-6935/79/244206-04\$00.50/0.

(c) 1979 Optical Society of America. method, described in Sec. II, uses the combination of a spatial modulation technique ${ }^{9-13}$ to synthesize incoherent bipolar pointspread functions (this technique is sometimes referred to as two-pupil interaction) and a multiplexing scheme using carrier frequencies with several orientations (i.e., theta modulation) ${ }^{14}$ Experimental results are presented in Sec. III together with a comparison with the results of a similar technique using coherent bandpass filtering for the encoding step.

\section{Description of the Method}

Compared with coherent optical systems using, for example, multiwavelength laser sources, optical processors with polychromatic light sources seem better suited for color encoding. All the colors of the source spectrum are available and can be isolated either by diffraction or color filtering. Furthermore, spatially incoherent optical systems have, theoretically, a much superior SNR. Incoherent processing, however, is plagued by the constraint that intensity (or irradiance) can only take real positive values. Operations such as high-pass or bandpass filtering, which require a bipolar pointspread function, can only be performed, with incoherent light, by either a multichannel system ${ }^{15-17}$ or a modulation technique using a spatial ${ }^{10-12}$ or a temporal ${ }^{9}$ carrier frequency.

A temporal carrier has the advantage that the full space-bandwidth product of the optical system is available for information transfer. The demodulation, however, requires a scan of the output followed by electronic signal processing. The use of a spatial carrier reduces the available space-bandwidth product by at least a factor of 2 . The most convenient spatial demodulation technique is probably also a scanning followed by a temporal high-pass filtering. It is also possible to record the spatially modulated output on film and to demodulate it in a spatial filtering setup. This last technique, for its convenience and availability, was 


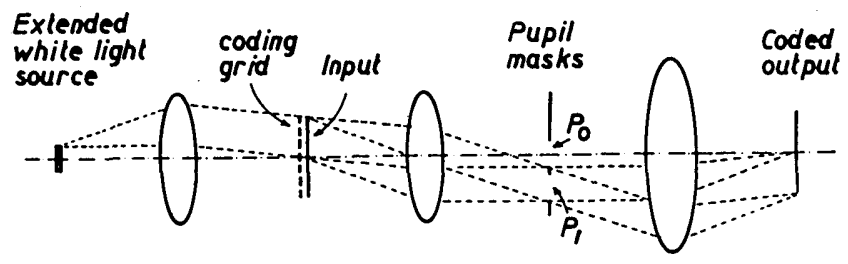

Fig. 1. Incoherent optical processor using spatial carrier modulation.

used for the experiments described here. The possibility of bypassing the intermediate photographic recording step by using a real-time spatial light modulator, a feature that would make the technique much more attractive, has been recently suggested. ${ }^{18}$

The optical arrangement used to modulate the input is shown in Fig. 1. It is a classical double diffraction system using an extended incoherent source. In the first stage, the input is either imaged on or put in contact with a Ronchi grid of fundamental frequency $u_{0}$. Each diffraction order of the grid projects a spatially isolated image of the source and of the object spectrum in the Fourier plane. These are made to interact independently with different pupil masks. If, for example, the zero order and the +1 order interact with, respectively, a pupil mask $P_{0}(u)$ and another $P_{1}\left(u-u_{0}\right)$, the system may be described as an incoherent imaging system with a transfer function equal to the autocorrelation of the pupil function, i.e.,

$$
\begin{aligned}
H(u)= & P_{0}(u) * P_{0}^{*}(-u)+P_{1}(u) * P_{1}^{*}(-u) \\
& +\left[P_{0}(u) * P_{1}^{*}(-u)\right] * \delta\left(u-u_{0}\right) \\
& +\left[P_{1}(u) * P_{0}^{*}(-u)\right] * \delta\left(u+u_{0}\right),
\end{aligned}
$$

where $*$ is short notation for the convolution product. Similarly, the intensity pointspread function can be written as

$$
\begin{aligned}
h(x)= & \left|p_{0}(x)\right|^{2}+\left|p_{1}(x)\right|^{2} \\
& +\left|p_{0}(x)\right|\left|p_{1}(x)\right| \cos \left(2 \pi u_{0} x+\phi_{1}-\phi_{0}\right),
\end{aligned}
$$

where the lowercase and corresponding uppercase letters are Fourier transform pairs, and $\phi_{0}, \phi_{1}$ are the phases of $p_{0}$ and $p_{1}$.

This development assumes that a few simplifying conditions are met. For example, there is an exact match of the pupil separation with the diffraction orders of the grid; the different diffraction orders do not overlap; the image of the source in the Fourier plane covers the entire area of the masks; etc. These conditions are not difficult to meet experimentally and will not be discussed further.

From Eqs. (1) and (2), it is clear that, even with simple binary (transparent and opaque) pupil masks, a wide range of bipolar pointspread functions, riding on a spatial carrier $u_{0}$, can be synthesized.

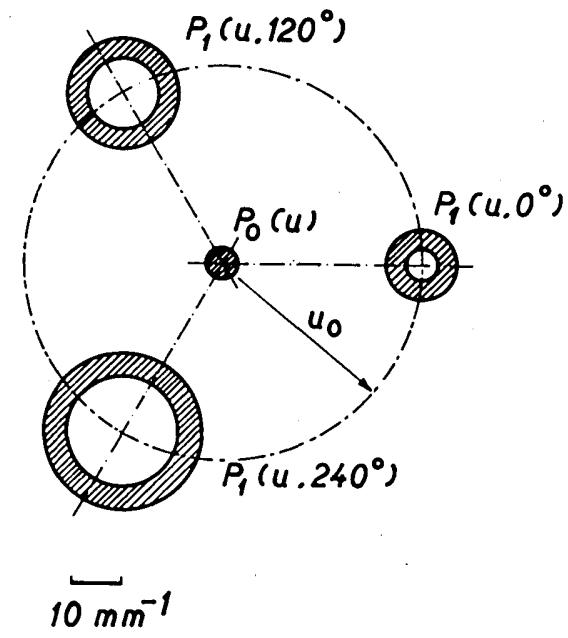

Fig. 2. Three angularly separated pupil masks corresponding to three incoherent bandpass filters.

\section{Experimental Results}

Three different sets of pupil masks were used to synthesize three bandpass filters. The masks are shown in Fig. 2. For this experiment, the three outputs were recorded sequentially on the same film frame, after orienting the grid so that the first diffraction order fell on the ring pupil, the smaller disk being centered on the zero order. For each set of pupil masks (the central disk and one ring), the output is a superposition of a dc image associated with a transfer function proportional to the autocorrelation of each mask and an ac image riding on a spatial carrier frequency $u_{0}$. The transfer function of the modulated image is proportional to the crosscorrelation of the two masks. Several images, processed with different transfer functions, can be multiplexed by using different orientations of the carrier (theta modulation).

The transfer functions for the three modulated images are shown in Fig. 3. They correspond to three bandpass filters with an approximate width of $6 \mathrm{~mm}^{-1}$, centered, respectively, on a spatial frequency of $5 \mathrm{~mm}^{-1}$, $9 \mathrm{~mm}^{-1}$, and $13 \mathrm{~mm}^{-1}$. The carrier frequency of the modulating grid was approximately $40 \mathrm{~mm}^{-1}$.

The demodulation setup sketched in Fig. 4 is another double diffraction arrangement in which the diffracted spectrum of each modulated image (the spectra are spatially separated in the Fourier plane) is coded by a color filter. 


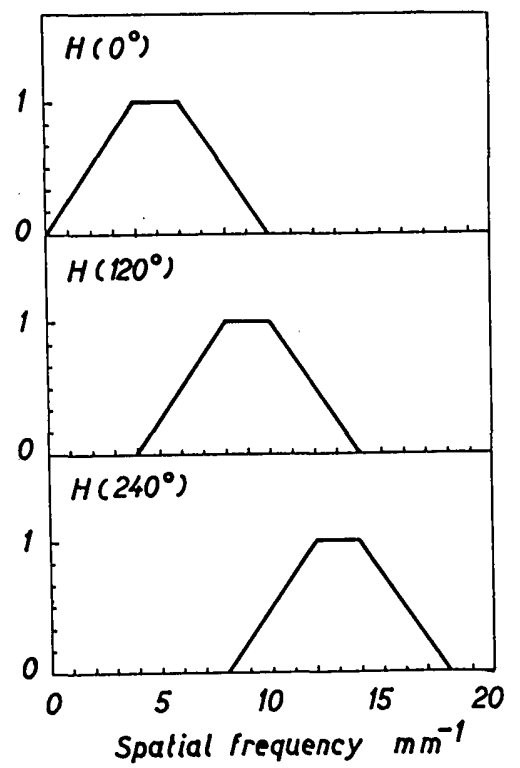

Fig. 3. Transfer functions of the three bandpass filters.

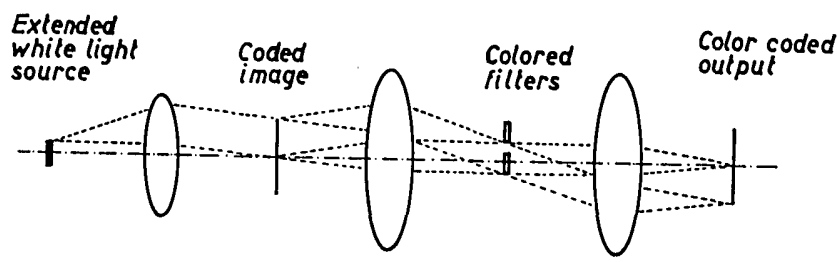

Fig. 4. Demodulation and color coding in a double diffraction setup using incoherent light.
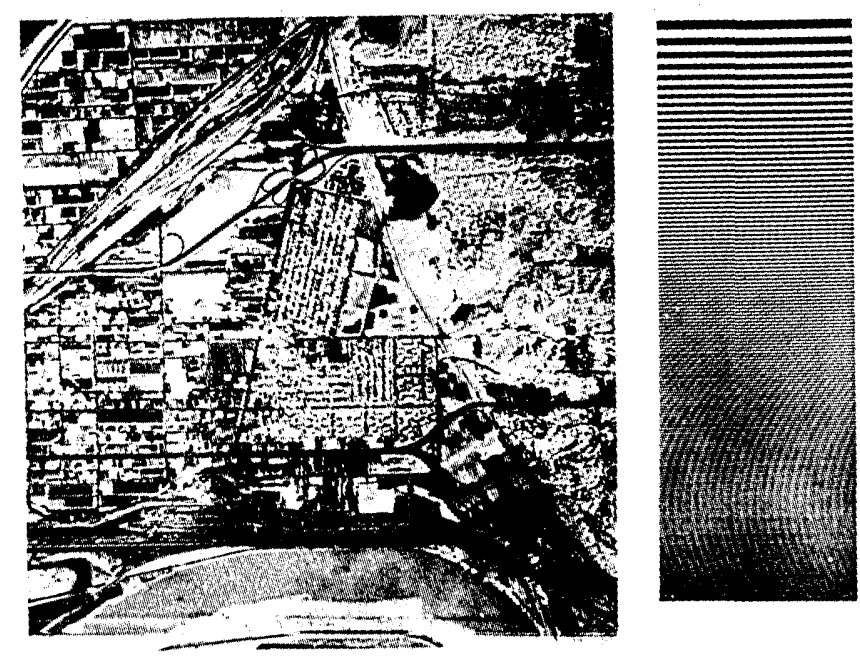

Fig. 5. Aerial photograph used as input.
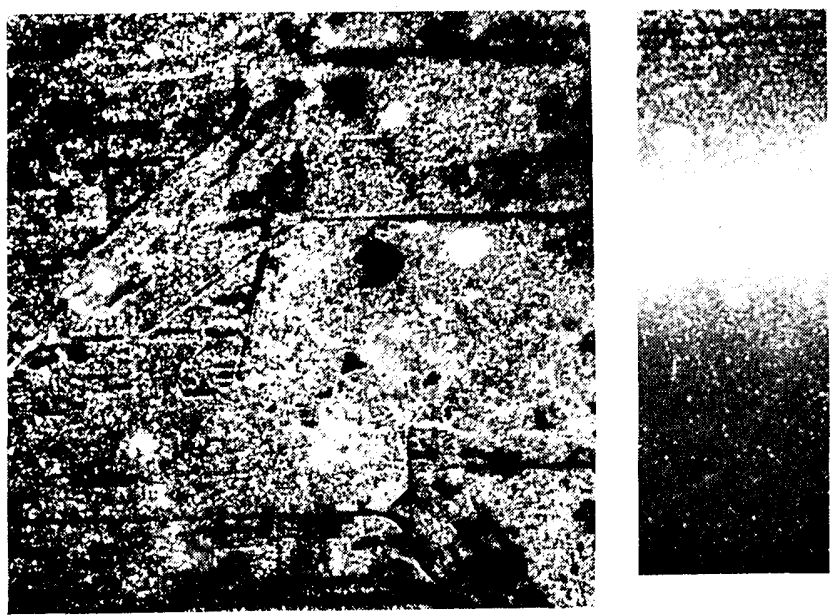

a
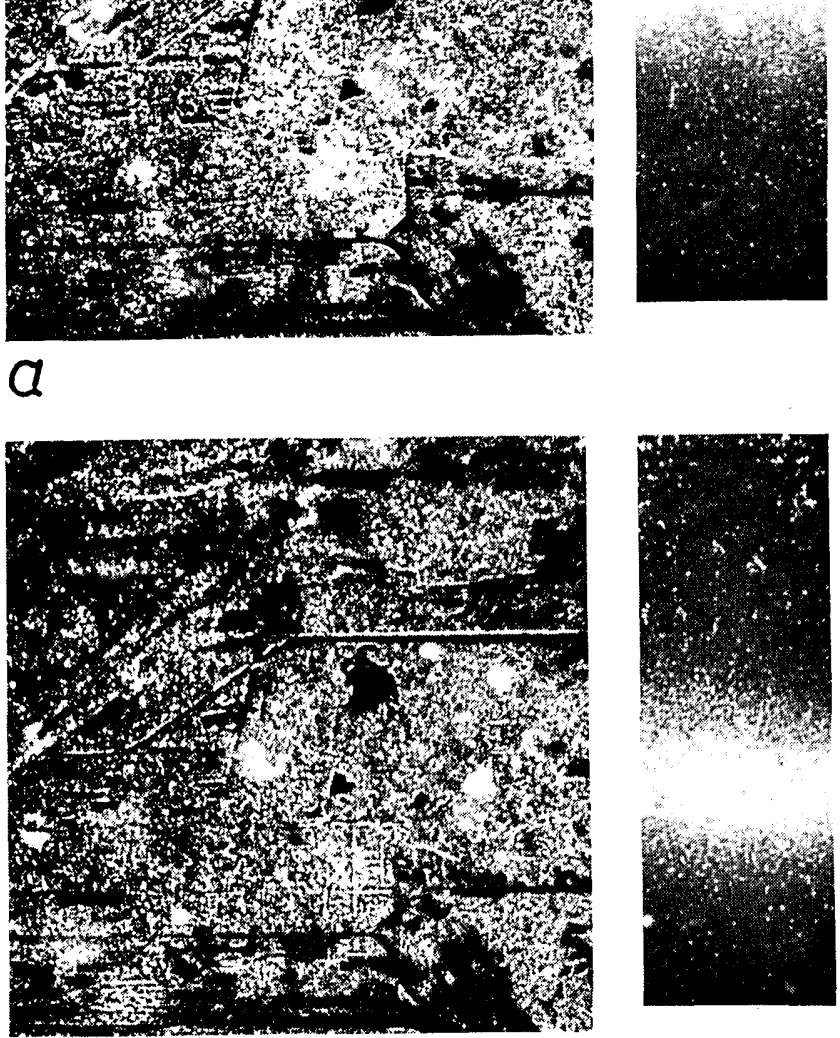

b
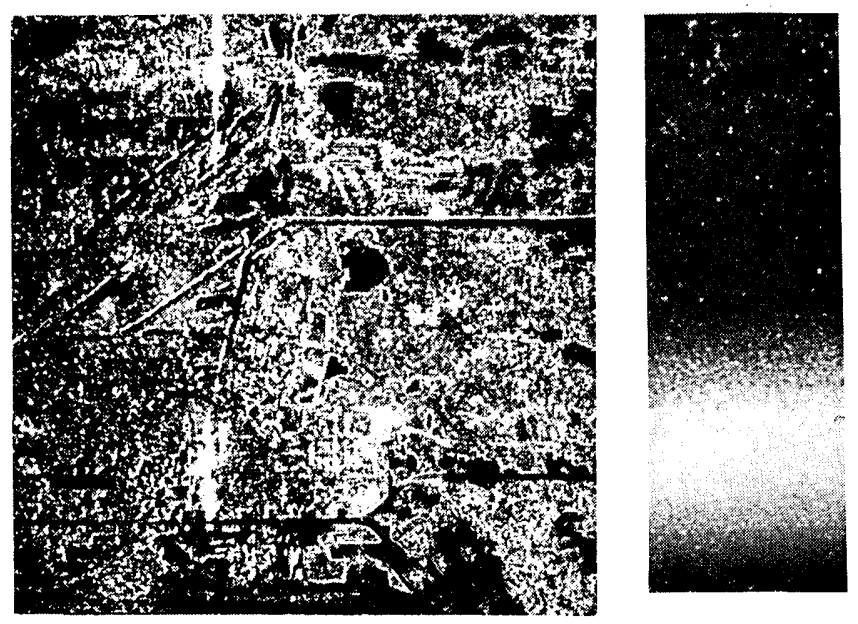

C

Fig. 6. Demodulated outputs corresponding to the bandpass filters of Fig. 3. All bands are $6 \mathrm{~mm}^{-1}$ wide and are centered on a spatial frequency: (a) $5 \mathrm{~mm}^{-1}$; (b) $9 \mathrm{~mm}^{-1}$; (c) $13 \mathrm{~mm}^{-1}$. 


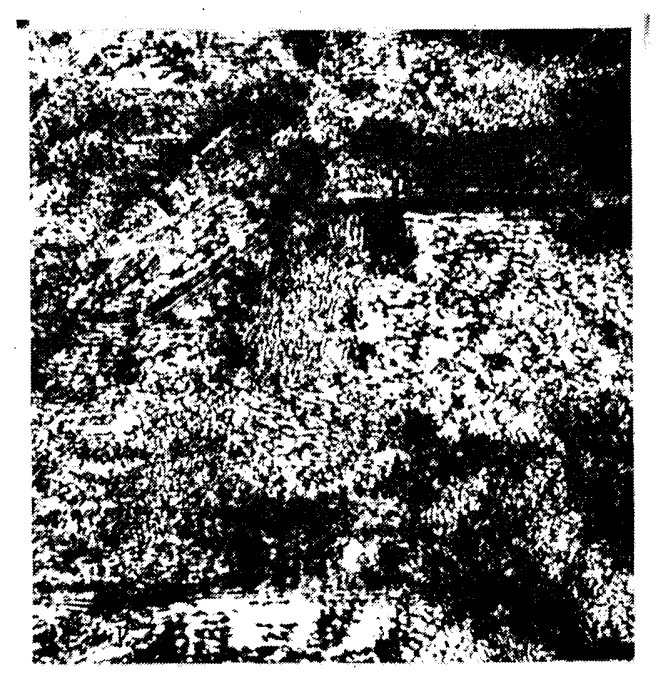

Fig. 7. Output corresponding to a bandpass filter centered on a frequency of $5 \mathrm{~mm}^{-1}$ recorded in coherent light.

Figure 5 shows an aerial photograph used as the input. The three demodulated images corresponding to the bandpass filters of Fig. 3 are shown in Fig. 6 . Note, for example, how each filter picks out different information in the periodical structures near the center of the picture. The output of the system of Fig. 4 is, of course, a colored picture in which the three images shown in Fig. 5 are superposed and coded in different colors.

The high level of noise in these pictures is due to the low modulation depth of the coded images. (The modulated pictures are superposed to a high dc background.) This results in a low diffraction efficiency and a poor SNR in the filtered output. In view of these results, one might wonder if the advantage of a higher SNR, which is often claimed for incoherent (vs coherent) optical processing is justified. Bandpass filtering, after all, is so much easier to perform in coherent light where the pupil function itself is the system transfer function. To answer this question, the same experiment was performed using coherent light to filter out three bands of spatial frequencies from the same input (Fig. 5) and code them with carrier fringes of different orientations. Figure 7 is the best output (corresponding to the lowest spatial frequency band) obtained with coherent encoding. The demodulation setup of Fig. 4 was the same for both experiments.

\section{Conclusions}

A technique using incoherent pupil interaction and theta modulation for color coding the spatial frequency content of an input image has been described. The coding of spatial frequencies is of particular interest in texture analysis and differentiation, a very important technique used, for example, in terrain recognition, analysis of aerial imagery, or feature extraction in radiography.
The experimental results obtained clearly demonstrate, at least for this application, the superiority of incoherent over coherent processing. Equally important is the fact that incoherent processing is, in principle, applicable to the direct imaging of incoherently illuminated objects, a feature not at all possible with coherent imaging.

The two-step technique used in this experiment is probably not the most practical for implementing the described transformation. Ideally, real-time modulation and demodulation using, for example, a spatial light modulator with a sharply peaked response near the spatial carrier frequency used for coding, would be highly desirable. Scanning of the output (using a TV camera or mechanical deflectors), followed by electronic processing of the video signal, would probably be the next most practical demodulation scheme.

\section{References}

1. H. K. Liu and T. W. Goodman, Nouv. Rev. Opt. 7, 285 (1976).

2. G. Indebetouw, Appl. Opt. 16,1951 (1977).

3. G. Indebetouw, J. Opt. 9, 1 (1978).

4. A. Tai, F. T. S. Yu, and H. Chen, Opt. Lett. 3, 190 (1978).

5. F. T. S. Yu, A. Tai, and H. Chen, J. Opt. 9, 269 (1978).

6. F. T. S. Yu, Opt. Lett. 3, 57 (1978).

7. P. N. Tamura, Appl. Opt. 17, 2532 (1978).

8. J. Bescos and T. C. Strand, Appl. Opt. 17, 2524 (1978).

9. W. T. Rhodes, Appl. Opt. 16, 265 (1977).

10. A. W. Lohmann, Appl. Opt. 16, 261 (1977).

11. W. Stoner, Appl. Opt. 16, 1451 (1977).

12. W. Stoner, Appl. Opt. 17, 2454 (1978).

13. A. W. Lohmann and W. T. Rhodes, Appl. Opt. 17, 1141 (1978).

14. J. D. Armitage and A. W. Lohmann, Appl. Opt. 4, 399 (1965).

15. D. Görlitz and F. Lanzl, Opt. Commun. 28, 283 (1979).

16. P. Wiersma, Opt. Commun. 28, 280 (1979).

17. P. Chavel and S. Lowenthal, J. Opt. Soc. Am. 66, 14 (1976).

18. A. Armand, A. A. Sawchuk, T. C. Strand, D. Bosswell, and B. H. Stoffer, in Proceedings ICO-11, Madrid, Spain, (1978), p. 253. 